\title{
Abdominal Muscular Metastases of a Pulmonar Adenocarcinoma
}

\author{
Joana de Castro Rocha1 ${ }^{*}$, Pedro Nuno Brandão², Ana Rita Cruz ${ }^{1}$, Vítor Valente ${ }^{2}$ \\ ${ }^{1}$ Internal Medicine Department, Centro Hospitalar do Porto, Porto, Portugal \\ ${ }^{2}$ General Surgery Department, Centro Hospitalar do Porto, Porto, Portugal \\ Email: ${ }^{*}$ joanadecastro.r@gmail.com
}

Received 11 June 2015; accepted 27 June 2015; published 30 June 2015

Copyright (C) 2015 by authors and Scientific Research Publishing Inc.

This work is licensed under the Creative Commons Attribution International License (CC BY).

http://creativecommons.org/licenses/by/4.0/

(c) (i) Open Access

\begin{abstract}
Metastases are synonymous of malignant neoplasm in advanced stage and so associated with a poor prognosis. As the first manifestation of cancer disease, the muscle metastasis is very rare. The definitive diagnosis of its presence is made based on histology. The authors presented the case of a patient with a lung adenocarcinoma, being with first manifestation of it, a muscle metastasis only about a month later there were clinical manifestations with imaging appearance that led to the diagnosis of a primary neoplasm. The rare manifestation of the disease motivated the study and surveillance of the patient and lead to the diagnosis.
\end{abstract}

Keywords

Muscular, Metastasis, Adenocarcinoma, Lung

\section{Introduction}

The lung cancer is the second leading cause of cancer both in men and women [1]. After the diagnosis of a malignant neoplasm of the lung, there are studies to stage the disease and, therefore, search for metastases. The most commonly found metastases are located in the brain, bones, adrenal gland, liver, contralateral lung and lymph nodes [2]-[4]. Other metastatic sites as skeletal muscle are extremely rare [1] [2], and there are very few cases described in literature [3] [4]. Some of these muscle metastases are diagnosed only when the autopsy is performed, since they are mostly asymptomatic [5]. Several factors have been proposed to explain the rarity of this type of metastases, such as blood flow in the muscle tissue, the type of cell metabolism, trauma and muscle contraction. It appears that these factors also contribute to the fact that they are diagnosed at different time intervals compared to the diagnosis of the primary neoplasm [1].

"Corresponding author. 
The most common sites for the onset of muscle metastases are the trunk muscles, particularly the muscles of the vertebral gutters and psoas [1]. There are reports of pulmonary adenocarcinoma metastases in the large buttock muscle [3], a case of metastasis in the pectoralis major muscle and another in the oculomotor muscles.

Clinically, the symptoms that often accompany this type of metastases are pain and a palpable swelling [3]. However, they may simply be asymptomatic.

As the muscle metastasis is a rare form of presentation of a primary malignant neoplasm of unknown origin, there is no clearly defined consensus on how to approach this type of metastasis. Options include a first approach by radiotherapy, chemotherapy or simply local excision, the latter is the most widely accepted [3].

\section{Case Presentation}

The authors present the case of a 52-year-old man, chronically dependent by a severe sensorimotor axonal neuropathy, with chronic liver disease caused by alcoholism, and active smoking with pulmonary chronic obstructive disease.

He went to the Emergency Service in September 2013 with severe abdominal pain in the lower right quadrant. The physical examination trough palpation caused increased pain with muscular defense but there were no palpable swellings and no other signals of peritoneal irritation. An abdominal computed tomography revealed a lesion of elongated morphology, with solid appearance, with emphasis on the administration of intravenous contrast, especially on the periphery of the lesion, located on the thickness of the muscles of the right anterolateral abdominal wall, in dependence of the transverse muscle; the average lesion was $39 \times 14 \mathrm{~mm}$ of larger transverse and anteroposterior diameter (Figure 1).

It was decided an elective surgical approach of the lesion (wide excision). This was determined, due to the fact that the lesion was of unknown etiology, but very symptomatic. The histopathological study revealed that it was a poorly differentiated carcinoma metastasis with positivity for CK 7 and CAM 5.2 and negative for TTF1, CK20 and Vimentin.

One month later, while studies in order to search for the primary tumor were in progress, the patient appealed again to the hospital Emergency Service because of a pain in the right inframammary region with irradiation to the back area of the right shoulder scapula and the cervical spine. A pulmonary computed tomography revealed a nodular lesion in the right hemithorax, solid with $4 \mathrm{~cm}$ in diameter, adjacent to the posterior arch of the fourth right rib with cortical erosion (Figure 2). The pulmonary radiography performed weeks before was considered normal.

The patient was admitted for the study of the nodule and pain control. A transthoracic biopsy with ultrasound of the nodule was performed, and the histological characterization revealed the presence of an adenocarcinoma.

Given the overall condition of the patient, it was decided a symptomatic treatment requiring opioid therapy. The patient died months later.

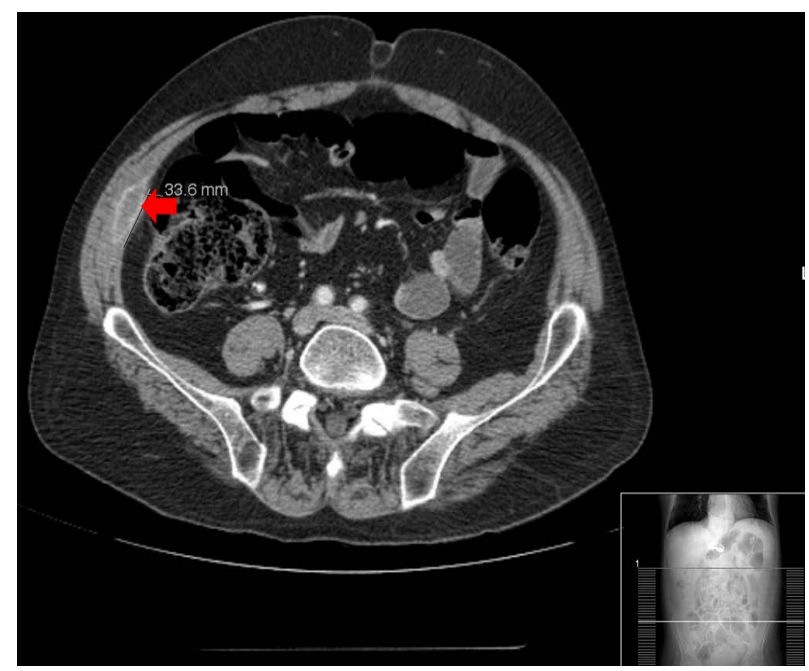

Figure 1. Abdominal computed tomography (arrow). 


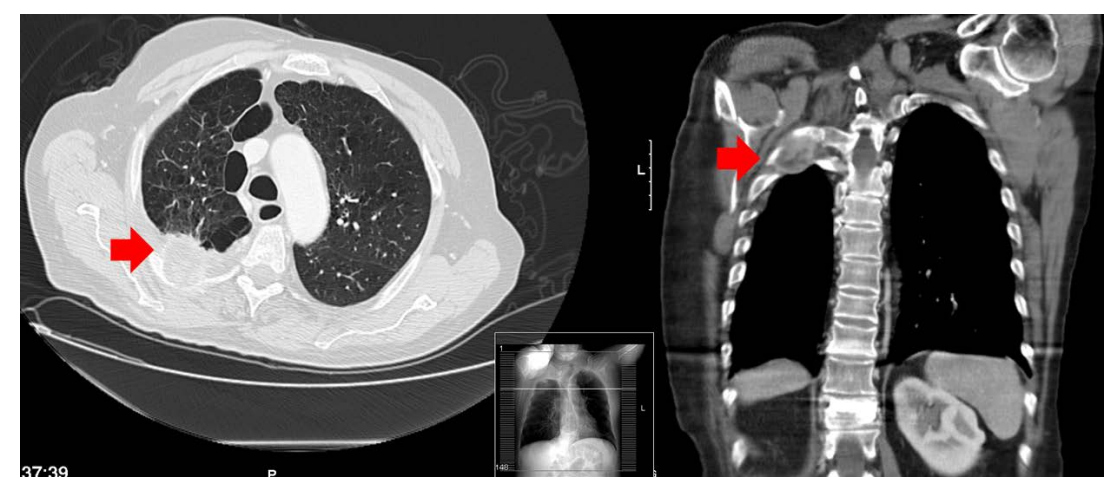

Figure 2. Pulmonary computed tomography (arrow).

\section{Discussion}

Positivity for CK7 appears to be related to the presence of a pulmonary adenocarcinoma, as seen in the histological piece of the reported clinical case, which is consistent with the diagnosis established by biopsy of the lung nodule.

Being the presentation of this oncological disease a muscle metastasis gives special interest to the clinical case by the rarity of this form of presentation.

The importance of the timely follow-up of the patients and the clinical surveillance of the symptoms, as well as the insight into the patient approach, make the study more directed to the search for the correct diagnosis.

The case presented here, with smoking as a major risk factor for pulmonary adenocarcinoma, had muscle metastases as a primary manifestation of his oncological disease. About one month later, a pulmonary nodule compatible with an adenocarcinoma was diagnosed. Given the co-morbidities and the advanced stage of the disease, it was proposed a symptomatic treatment, according to the literature.

\section{References}

[1] Giugliano, F.M., Alberti, D., Guida, G., de Palma, G., Iadanza, L., Mormile, M., Cammarota, F., Montanino, A., Fulciniti, F., Ravo, V. and Muto, P. (2013) Non Small-Cell Lung Cancer with Metastasis to Thigh Muscle and Mandible: two Case Reports. Journal of Medical Case Reports, 7, 98. http://dx.doi.org/10.1186/1752-1947-7-98

[2] Strauss. J.B., Shah, S.S., Chen, S.S., Gielda, B.T. and Kim, A.W. (2011) Psoas Muscle Metastasis in Non-Small Cell Lung Cancer. Journal of Thoracic Disease, 4, 83-87.

[3] Purandare, N.C., Ranagarajan, V., Pramesh, C.S., Rajnish, A., Shan, S. and Dua, S.G. (2008) Isolated Asymptomatic Skeletal Muscle Metastasis in a Potencially Resectable Non-Small Cell Lung Cancer: Detection with FDG PET-CT Scanning. Cancer Imaging, 8, 216-219. http://dx.doi.org/10.1102/1470-7330.2008.0033

[4] Tezcan, Y. and Koc, M. (2014) Muscle Metastasis from Non-Small Cell Lung Cancer: Two Cases and Literature Review. Acta Clinica Belgica, 69, 302-304.

[5] Kwas, H.H., Zendah, I. and Ghedira, H. (2013) Skeletal Muscle Metastasis from Lung Cancer. Asian Cardiovascular and Thoracic Annals, 21, 741-743. http://dx.doi.org/10.1177/0218492312470571 